\title{
TARDE DO SABER
}

\section{AFTERNOON LEARNING}

Gabriela Giro1', Jenifer Toledo Damasceno², Juliana Andrade Oliveira Queiroz ${ }^{3}$, Nathalia Freitas Figueiredo4, Belén Stephanie Retamal-Valdés ${ }^{5}$, Marina Guimarães Roscoe ${ }^{6}$, Magda Feres ${ }^{7}$ Luciene Cristina Figueiredo ${ }^{8}$, Murilo Neuppmann Feres ${ }^{9}$, Bruno Bueno-Silva ${ }^{10}$

\section{RESUMO}

O objetivo deste artigo é relatar o projeto Tarde do Saber, desde sua concepção até sua execução. A Tarde do Saber foi planejada como uma ação conjunta de três programas de pós-graduação da Universidade Univeritas UNG: Programa de Pós-Graduação em Odontologia, Enfermagem e Geociências. O envolvimento dos três programas foi considerado importante, pois cada programa poderia transmitir diferentes áreas de conhecimentos, atraindo, assim, um público diverso. Desta maneira, a intenção foi promover um contínuo interesse dos professores do ensino fundamental em ciência, pois sabe-se que uma maneira de evoluir o país (em todos os sentidos) é estimular o desenvolvimento da ciência neste país, porém uma frustrante experiência foi encarada, uma vez que não houve interesse por parte desses professores em participar deste projeto. Frente a este resultado negativo, optou-se por realizar a Tarde do Saber, como uma atividade dentro das Aulas de Trabalho Pedagógico Coletivo (ATPC) promovido pela Diretoria de ensino regional de Guarulhos, SP, cujo objetivo é consolidar as práticas pedagógicas previstas pela Secretaria de Educação assim como o aperfeiçoamento dos docentes com vistas às avaliações para qualificação e evolução profissional. Além disso, somente o Programa de Pós-graduação em Odontologia continuou com a atividade. Esta experiência mostrou que a atividade TARDE DO SABER pode contribuir para a formação continuada dos docentes da educação básica e assim, pode-se contribuir para a constante preocupação sobre a formação possivelmente deficiente dos docentes da educação básica.

PALAVRAS-CHAVE: Formação continuada. Educação Básica. Educação científica.

\footnotetext{
Professor do programa de Pós graduação em Odontologia da Universidade UNG, Guarulhos, SP.

Aluno do curso de graduação em Odontologia da Universidade UNG, Guarulhos, SP.

Aluno do curso de Pós graduação em Odontologia da Universidade UNG, Guarulhos, SP.

Aluno do curso de Pós graduação em Odontologia da Universidade UNG, Guarulhos, SP

Professor do programa de Pós graduação em Odontologia da Universidade UNG, Guarulhos, SP.

Professora do Departamento de Biomateriais e Biologia Oral da Faculdade de Odontologia da USP (FOUSP)

Professor do programa de Pós graduação em Odontologia da Universidade UNG, Guarulhos, SP.

Professor do programa de Pós graduação em Odontologia da Universidade UNG, Guarulhos, SP.

Professor do Departamento de Clínica Infantil, Faculdade de Odontologia de Ribeirão Preto, USP.

10 Professor do programa de Pós graduação em Odontologia da Universidade UNG, Guarulhos, SP.
} 


\section{ABSTRACT}

The purpose of this article is to report the Afternoon Learning project, from its conception to its execution. Afternoon Learning was planned as a common action of three graduate programs of UNG Univeritas University: Graduate Program in Dentistry, Nursing and Geosciences. The involvement of the three programs was considered important because each program could transmit different areas of knowledge, thus attracting a diverse audience. In this way, the intention was to promote a continuous interest of teachers from fundamental education in science, since it is known that one way of improving the country (in all the senses) is to stimulate science development. However, a frustrating experience was envisaged, since there was no interest on the part of these teachers to participate in this project. Faced this negative result, it was decided to hold the Afternoon Learning as an activity within the Collective Pedagogical Work Classes (CPWC) promoted by the Regional Education Board of Guarulhos, SP, whose objective is to consolidate the pedagogical practices envisaged by the Education Department as well as the teachers improvement in the light of qualification evaluation and professional evolution. In addition, only the Postgraduate Program in Dentistry continued with the activity. This experience has shown that the Afternoon Learning project can contribute to the continuous education of basic education teachers and thus, can contribute to the constant concern about the possibly deficient formation of the teachers from basic education.

KEYWORDS: Continuing education. Basic education. Science education

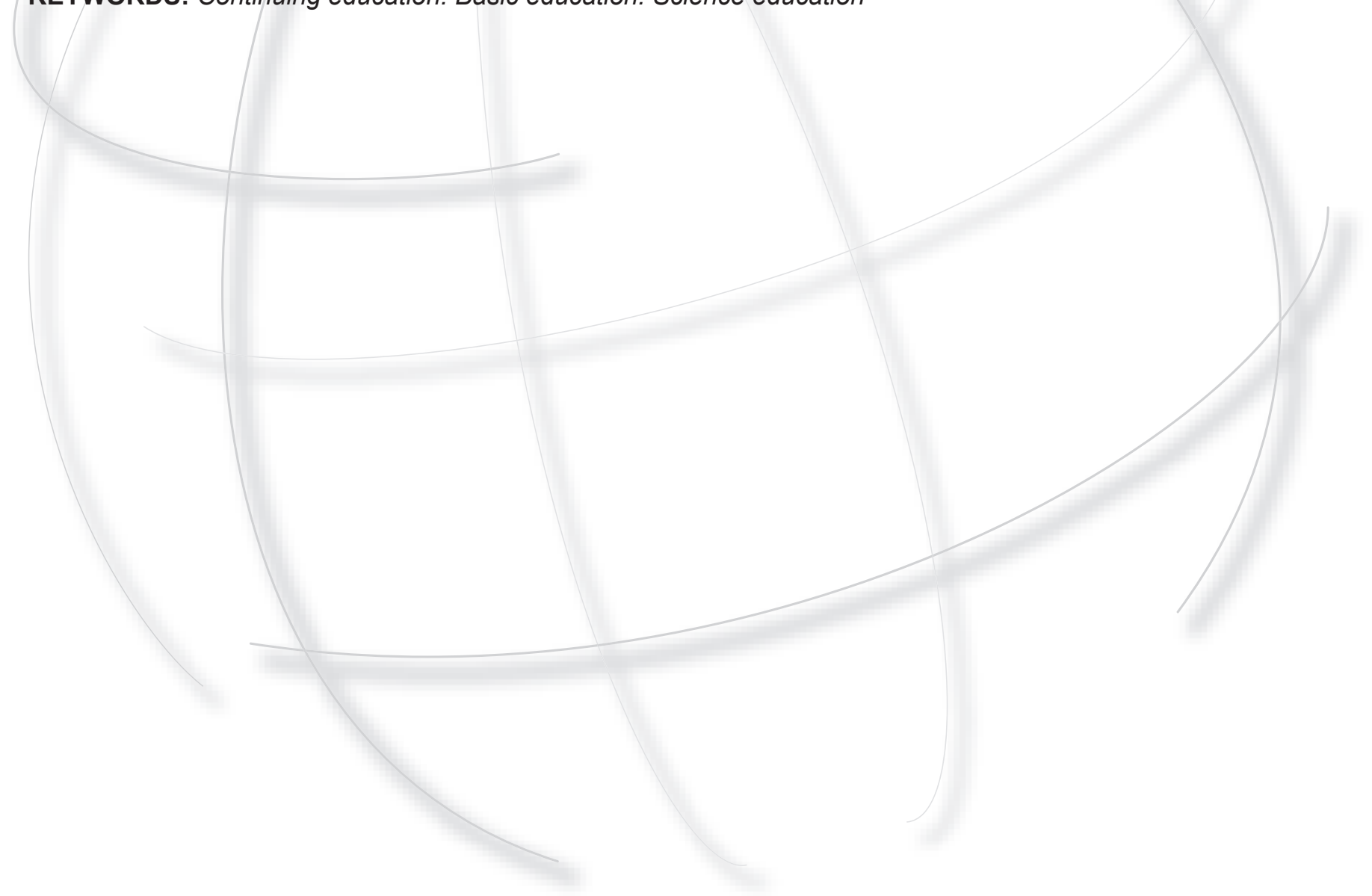




\section{INTRODUÇÃO}

A educação pode ser definida como um processo onde pessoas, com graus variáveis de conhecimento acerca de um tema, se propõem a compartilhar este conhecimento com pessoas de menor conhecimento a respeito deste mesmo ${ }^{1}$. Em se tratando de educação escolar, o professor assume um papel central neste processo, onde ele é o responsável por transmitir conhecimentos para os demais. Assim, a qualidade da formação dos professores é fundamental para a completa e adequada formação dos estudantes de um país. (GATTI, 2016; LONGHINI, 2008; SELLES; ANDRADE, 2003).

Para se avaliar a qualidade da formação dos professores, deve-se levar em consideração alguns aspectos tais como: conhecimento da cultura geral, suas atitudes em sala de aula, sua formação tanto cultural como profissional e seu material didático é de fundamental importância para a transmissão do conhecimento (GATTI, 2016; LONGHINI, 2008). Neste sentido, a preocupação com a qualidade da formação dos professores é antiga e constante não só pelas autoridades competentes, mas também por toda a população que depende desses profissionais para sua própria educação e/ou educação de seus filhos. (GATTI, 2016; LONGHINI, 2008; SELLES; ANDRADE, 2003; FREITAS, 1998; CARVALHO, 2003; MARIN, 2003).

Entretanto, sabe-se que a qualidade da formação inicial dos professores do ensino fundamental não é a mais adequada para a realidade socioeconômica do nosso país e também para a realidade encontrada em sala de aula (SELLES; ANDRADE, 2003). Neste contexto, a literatura demonstra que os docentes apresentam deficiência no domínio e conhecimento do tema que deve ser transmitido aos alunos, não proporcionando assim a correta educação dos alunos, os quais são os maiores prejudicados (GATTI, 2016; LONGHINI, 2008; SELLES; ANDRADE, 2003; FREITAS, 1998; CARVALHO, 2003; MARIN, 2003).

Uma possível solução para esta deficiência pode ser a formação continuada, processo no qual os professores estão sendo continuamente formados, e este processo pode ser considerado uma peça fundamental para melhoria da atuação docente como transmissor de conhecimento (NÓVOA, 1995). Além disso, Bonzanini e Bastos (2013) apontam a formação continuada como solução para suprir as lacunas deixadas pela formação inicial dos docentes.

Tendo em vista a formação continuada dos professores, o programa de Pós-Graduação em Odontologia da Universidade Univeritas UNG criou o projeto de extensão intitulado "Tarde do Saber", que visa contribuir para a formação continuada dos docentes do ensino fundamental da rede pública de Guarulhos, no estado de SP, (Brasil), por meio da transmissão dos conhecimentos gerados em seus laboratórios de pesquisa, para os docentes do ensino fundamental e médio, em uma linguagem acessível para que estes possam aproveitar tal conhecimento e transmiti-lo a seus respectivos alunos.

Assim, o objetivo deste artigo é relatar o projeto Tarde do Saber, desde sua concepção até sua execução.

\section{RELATO DE EXPERIÊNCIA}

\section{Planejamento inicial da "Tarde do Saber"}

Inicialmente, a Tarde do Saber foi planejada como uma ação conjunta de três programas de pós-graduação da Universidade Univeritas UNG: Programa de Pós-Graduação em Odontologia, Enfermagem e Geociências. A ideia da Tarde do Saber era contribuir para maior conhecimento científico dos professores das escolas estaduais da região de Guarulhos, SP, de uma maneira que os mesmos possam utilizar o conhecimento adquirido na Tarde do Saber dentro de sala de aula e assim, transmitir estes conhecimentos científicos para seus alunos. Assim, o envolvimento dos três programas foi considerado importante, pois cada programa poderia transmitir diferentes áreas de conhecimentos, atraindo, assim, um público diverso, visto que a falta de conhecimento científico dos professores escolares está bem descrita na literatura, principalmente dos professores de ciências (LONGHINI, 2008). Desta maneira, a intenção e promover um contínuo interesse das crianças na ciência, pois sabe-se que uma maneira de evoluir o país (em todos os sentidos) é estimular o desenvolvimento da ciência neste país.

Para isso, foi feito contato com 21 escolas estaduais da cidade de Guarulhos, SP, Brasil, explicando o projeto Tarde do Saber e solicitando para que os respectivos coordenadores divulgassem tal ação entre os seus professores. Posteriormente, alguns professores do Programa de Pós-Graduação em Odontologia visitaram as escolas, conversaram com os coordenadores 
explicando e sanando eventuais dúvidas. Além disso, foram entregues diversos exemplares do folder (Figura 1) para divulgação nas escolas visitadas.

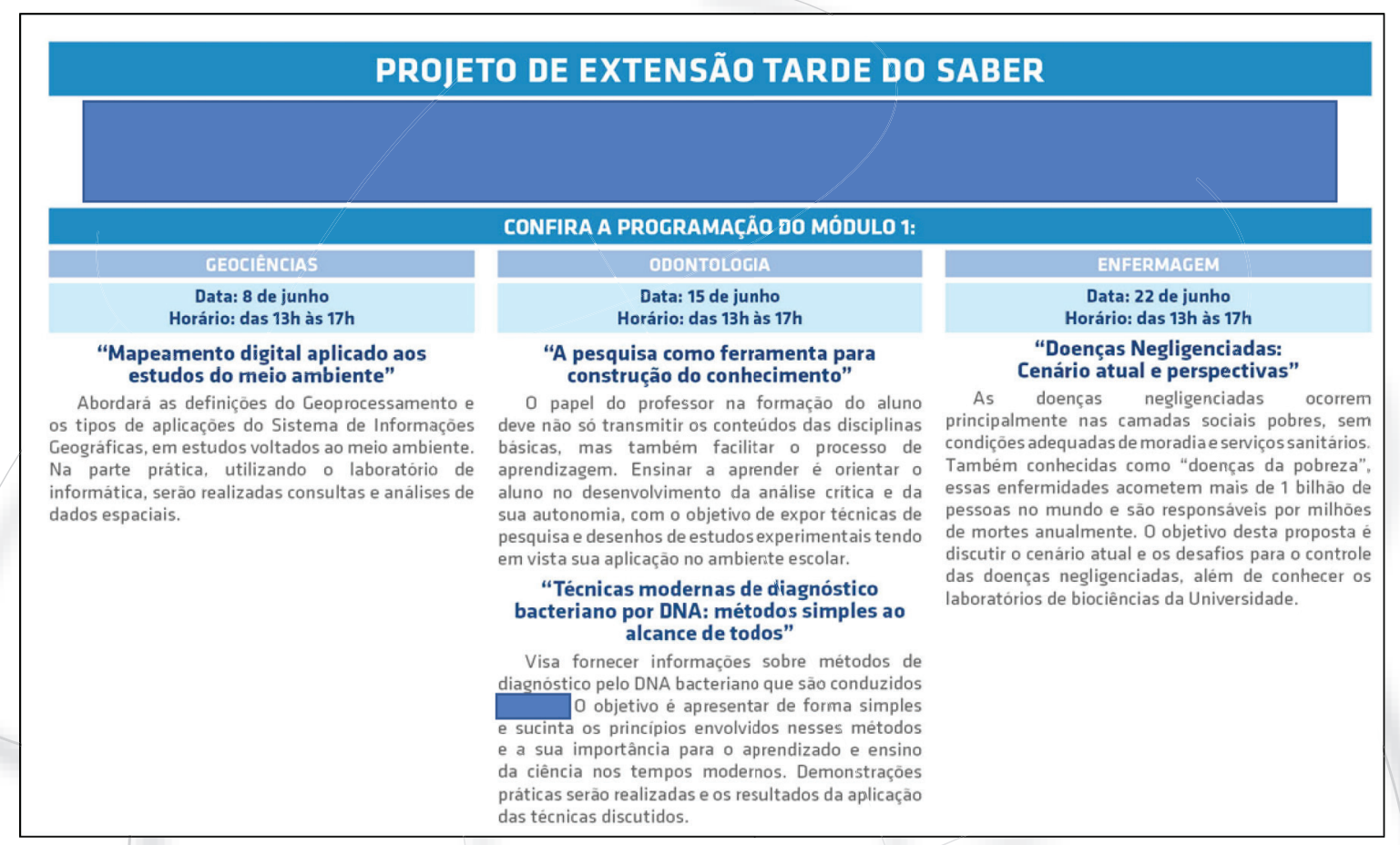

Figura 1: Folder de divulgação do Projeto de extensão Tarde do Saber entregue nas escolas estaduais

No entanto, apesar de todo o empenho e divulgação da atividade de extensão, não houve inscritos neste modelo de atividade. É importante ressaltar a falta de adesão inicial dos professores da educação básica ao processo de formação continuada proposto. Como possíveis explicações para essa falta de adesão foram levantadas as seguintes hipóteses: falta de tempo devido a extensa jornada de trabalho, a falta de interesse em melhorar sua própria formação, dificuldade de locomoção para o local proposto para a atividade, tema não relacionado a sua área de trabalho.

Frente a este resultado negativo, foram realizadas reuniões com coordenadores de duas das escolas que mostraram interesse em achar uma nova alternativa, e concluiu-se que realizar atividade fora do local de trabalho do professor não era uma opção atrativa. Neste sentido, optou-se por tentar novamente a realização da "Tarde do Saber", no entanto desta vez como uma atividade dentro das Aulas de Trabalho Pedagógico Coletivo (ATPC) promovido pela Diretoria de ensino regional de Guarulhos, SP, cujo objetivo é consolidar as práticas pedagógicas previstas pela Secretaria de Educação assim como o aperfeiçoamento dos docentes com vistas às avaliações para qualificação e evolução profissional.

\section{Tarde do Saber como uma ATPC}

Assim, tendo por base o contato com as diversas escolas estaduais da cidade de Guarulhos durante a divulgação da primeira tentativa do projeto, as duas escolas que demonstraram maior interesse e participação na divulgação do projeto foram selecionadas para a segunda tentativa do projeto. Assim, a aula "Produtos naturais utilizados em odontologia: estudos com própolis e curcumina" foi apresentada em dois dias diferentes em uma das escolas estaduais de forma que todos os professores, tanto os que trabalham no período matutino quanto no vespertino, pudessem assistir a mesma aula. Estas aulas foram propostas levando-se em consideração uma postagem recente em uma rede social, realizada por uma figura pública, bastante conhecida por suas receitas naturais, que afirmava que o uso da curcumina 
poderia substituir os dentifrícios fluoretados. Além disso, o uso da própolis como produto natural sempre desperta dúvidas. Dessa forma, os resultados científicos mais recentes em relação ao possível uso ou não destes produtos nos cuidados com a higiene oral foram apresentados de maneira que todos os presentes pudessem compreender. Nesta escola, cerca de 20 professores participaram das duas atividades. É importante relatar que após a aula, várias perguntas foram realizadas e acredita-se que foi possível sanar diversas dúvidas não apenas em relação ao tema proposto, mas também em relação a saúde bucal como um todo.

$\mathrm{Na}$ outra escola que demonstrou interesse em contar com as atividades da Tarde do Saber, foi ministrada a aula intitulada "Trauma dental, o que fazer?". Este tema foi escolhido pois a coordenadora relatou que, por coincidência, este fato havia ocorrido recentemente na escola e que os professores da escola ficaram em dúvida sobre qual a melhor conduta a ser adotada nestes casos. Assim, a aula apresentou claramente os procedimentos mais adequados a serem seguidos em caso de ocorrência de trauma dental. Mais uma vez, após a aula, os professores fizeram diversos questionamentos sobre a saúde bucal geral.

Por fim, uma outra vertente da Tarde do Saber incluiu instruções de higiene oral para os alunos de uma das escolas deste projeto, resultando na orientação de higiene oral para cerca de 140 crianças do sexto ano escolar, ensinando-os sobre a melhor maneira de se escovar os dentes. O fato da escola dispor de estrutura apropriada para esta atividade contribuiu sobremaneira para o sucesso da mesma. Além disso, o projeto contou com ajuda de uma indústria do ramo odontológico que doou kits de higiene bucal para as crianças.

\section{CONCLUSÃO}

Portanto, acredita-se que a atividade Tarde do saber contribui para a formação continuada dos docentes da educação básica e assim, pode-se contribuir para a constante preocupação sobre a formação possivelmente deficiente dos docentes da educação básica. 


\section{REFERÊNCIAS}

BONZANINI, T. K.; BASTOS, F. Estratégias de formação continuada de professores: análise de uma experiência. In: CONGRESO INTERNACIONAL SOBRE INVESTIGACIÓN EN DIDÁCTICA DE LAS CIÊNCIAS, IX, 2013, Girona, p. 448-453.

CARVALHO, A. M. P. A inter-relação entre Didática das Ciências e a Prática de Ensino. In: SELLES, SE..; FERREIRA, M.S. Formação docente em Ciências: memórias e práticas. Niterói: Eduff; 2003. p.117-135.

FREITAS, D. 0 aperfeiçoamento de professores em exercício no ensino de ciências: a quem interessam os resultados? 1998. Dissertação [Mestrado] São Carlos: UFSCar, 1998.

GATTI, B. A. Formação de professores: condições e problemas atuais. Revista Internacional de Formação de Professores. Itapetininga, SP, v. 1, n. 2, p.161-171, 2016.
LONGHINI, M. D. O conhecimento do conteúdo científico e a formação do professor das séries iniciais do ensino fundamental. Investigações em Ensino de Ciências. Porto Alegre, RS, v. 13, n. 2, p. 241-253, 2008.

MARIN, A. J. Formação de professores: novas identidades, consciência e subjetividade. In: TIBALLI E. F. A.; CHAVES, S. M. Concepções e práticas em formação de professores: diferentes olhares. Rio de Janeiro. DP\&A; 2003. p. 57-86.

NÓVOA, A. Os professores e a sua formação. 2. ed. Lisboa: Dom Quixote, 1995.

SELLES, S. E.; ANDRADE, E. P. Saberes docentes em formação: a pesquisa e a prática de ensino nas licenciaturas em Ciências Biológicas e História. Pro-Posições [online]. 2003; v. 24, n. 1, p. 109-112, 2003. 